\title{
Stochastic Analysis of the Eigenvalue Problem for Mechanical Systems Using Polynomial Chaos Expansion-Application to a Finite Element Rotor
}

\author{
E. Sarrouy ${ }^{1}$ \\ Postdoctoral fellow \\ e-mail: emmanuelle.sarrouy@ec-lyon.fr \\ 0. Dessombz \\ Lecturer \\ e-mail: olivier.dessombz@ec-lyon.fr \\ J.-J. Sinou \\ Professor \\ e-mail: jean-jacques.sinou@ec-lyon.fr \\ École Centrale de Lyon \\ Laboratoire de Tribologie et Dynamique \\ des Systèmes (UMR CNRS 5513), \\ 36 avenue Guy de Collongue, \\ 69134 Ecully, Cedex, France
}

\begin{abstract}
This paper proposes to use a polynomial chaos expansion approach to compute stochas-tic complex eigenvalues and eigenvectors of structures including damping or gyroscopic effects. Its application to a finite element rotor model is compared to Monte Carlo simulations. This lets us validate the method and emphasize its advantages. Three different uncertain configurations are studied. For each, a stochastic Campbell diagram is proposed and interpreted and critical speeds dispersion is evaluated. Furthermore, an adaptation of the Modal Accordance Criterion (MAC) is proposed in order to monitor the eigenvectors dispersion.
\end{abstract}

Keywords: stochastic, eigenvalue problem, polynomial chaos, rotordynamics, free vibration

\section{Introduction}

Introducing uncertainties in structures is now an acknowledged challenge. Variability may come from the manufacturing process leading to variations in the geometry or material properties, some deterioration or evolution of the structure during its lifetime. Several methods address the problem of introducing the uncertainty and propagating it in order to statistically characterize the system response, whether static or dynamic in case of an excitation or even its modal properties. The reader is referred to Ibrahim [1] and Ibrahim and Manohar [2] as well as Schuëller et al. successive reviews papers [3-5] for an extensive review of the literature. One may still shortly recall three of the main methods which use a parametric description of uncertainties. First, let us mention perturbation methods which are based on Taylor series expansion on a set of zero mean random variables; the series are usually truncated to first or second order because of computational difficulties. The present work relies on the second usual method that is the polynomial chaos (PC) expansion [6]: uncertain quantities are described by Hermite multivariate polynomials depending on standard normal variables. Further work extended this approach to other polynomials and is referred to as generalized Polynomial Chaos [7,8]. The third method is the well-known Monte Carlo (MC) simulation which consists in direct computation of the response for a large sample that lets the statistical indicators relative to the quantities of interest be rebuilt. Finally, let us mention another class of methods called non-parametric approaches [9] which rely on maximization of statistical entropy.

In this paper, we will focus on the eigenvalues and eigenvectors dispersion. The proposed method is exposed in view of an application to rotating structures to suit the treated example but can be applied to any linear system. This work aims at dealing with mechanical systems that include damping and/or gyroscopic effects. In these cases, complex eigenvalues and eigenvectors are to be considered and most of all, a possible separated evolution of real and imaginary parts of each quantity. Some older and very recent work deal with the stochastic eigenvalue problem: theoretical and practical considerations can be found in the book by Scheidt and Purkert [10], Ref. [11] compares performance of perturbation method, Polynomial Chaos expansion and direct Monte Carlo simulations for simple mass/springs systems, Ref. [12] examines the stochastic eigenmodes of a rotor with spatial random variation of its stiffness and density using a perturbation method, Refs. $[13,14]$ use a non-parametric approach to evaluate eigenmodes dispersion of rotors, and Ref. [15] extends perturbation approach to complex modes study. To the authors' knowledge, there is currently no work devoted to the eigenvalue problem of complex dynamical systems (i.e. methods which impose no condition on the mathematical properties of the matrices describing the structure) relying on the Polynomial Chaos method.

The current method consists in a generalization of what was developed in Ref. [16] where simple linear systems were described by a mass and a stiffness matrix only. As opposed to what is exposed in Ref. [11] the coefficients of the PC expansion are not evaluated based on MC sampling but using a Galerkin method on the normalized eigenvalue problem [17].

The next section is dedicated to the theoretical presentation of the method. It includes recalls on the eigenvalue problem in the case of rotating structures. Section 3 is devoted to the application to a finite element model of a rotor with three discs and two bearings. It details the structure and its equations as well as its deterministic study. Then, three different uncertain configurations are studied with multiple uncertain parameters in order to demonstrate efficiency and robustness of the proposed method. An adaptation of the Modal Accordance Criterion (MAC) to monitor the eigenvectors dispersion and a way to evaluate critical speeds dispersion with no further PC coefficients computation are presented too. 
A third subsection compares the results obtained by the proposed approach with direct MC simulations. It also addresses the issue of computation time and ways to improve efficiency. Finally, results on the three uncertain cases are presented and commented: eigenvalues dispersion is rendered using stochastic Campbell diagrams and critical speeds histograms while stochastic eigenvectors are monitored using the proposed stochastic MAC for different rotation speeds and more simple plots at critical speeds.

\section{Stochastic Theory for Eigenmodes using Polynomial Chaos Expansion}

This section is devoted to the presentation of the method. First, recalls on the deterministic eigenvalue problem in the context of rotating machinery are exposed. Then, uncertain parameters are introduced and the method providing the PC approximation coefficients is detailed.

2.1 Recalls on the Deterministic Eigenvalue Problem. When dealing with rotating machinery, the dynamic equations in the linear undamped deterministic case are written:

$$
\mathbf{M} \ddot{\mathbf{q}}+\mathbf{G} \dot{\mathbf{q}}+\mathbf{K q}=\mathbf{0}
$$

$\mathbf{q}, \dot{\mathbf{q}}$ and $\ddot{\mathbf{q}}$ denote the vector of degrees of freedom (dofs) and its first and second derivatives with respect to time. $\mathbf{M}$ and $\mathbf{K}$ are the usual mass and stiffness matrices. They are respectively definite symmetric positive and symmetric positive. $\mathbf{G}$ is the gyroscopic matrix which reflects effects of spatially distributed mass around rotation axis. It is skew-symmetric and proportional to the rotation speed $\Omega$. System size is denoted $n$.

To determine the eigenvalues and eigenvectors of this equation, the augmented formulation

$$
\mathbf{B} \dot{\mathbf{x}}-\mathbf{A x}=\mathbf{0}
$$

is generally used, where

$$
\mathbf{x}=\left\{\begin{array}{c}
\mathbf{q} \\
\dot{\mathbf{q}}
\end{array}\right\}, \quad \mathbf{A}=\left[\begin{array}{cc}
\mathbf{0} & \mathbf{K} \\
-\mathbf{K} & -\mathbf{G}
\end{array}\right] \quad \text { and } \quad \mathbf{B}=\left[\begin{array}{cc}
\mathbf{K} & \mathbf{0} \\
\mathbf{0} & \mathbf{M}
\end{array}\right]
$$

This augmented formulation leads to the following eigenvalue problem

$$
\mathbf{A} \mathbf{u}_{k}=\lambda_{k} \mathbf{B} \mathbf{u}_{k}
$$

Augmented eigenvectors are normalized with respect to $\mathbf{B}$ :

$$
{\overline{\mathbf{u}_{k}}}^{\mathrm{T}} \mathbf{B} \mathbf{u}_{k}=1
$$

This takes into account the complex nature of eigenvectors due to skew-symmetric matrix G. This way, the $2 n$ couples of eigenvalues and eigenvectors $\left(\lambda_{k}, \mathbf{u}_{k}\right)$ are uniquely defined.

In the considered case of undamped linear rotating systems, eigenvalues $\lambda_{k}$ are purely imaginary quantities. Their imaginary part will be denoted $\omega_{k}\left(\lambda_{k}=\mathbf{j} \omega_{k}\right)$. Eigenvectors are complex quantities but in the undamped case, they define a planar deformed shape.

Finally, in the field of rotating machinery, eigenvalues change with rotation speed due to gyroscopic matrix $\mathbf{G}$ dependency on $\Omega$. The Campbell diagram is then drawn to depict their evolution along rotation speed by plotting $\omega_{k}$ versus $\Omega$, as it provides a quick overview of the structure behavior [18].

2.2 Introducing Uncertainties. When some elements of the structure are uncertain, the dynamic system in Eq. (1) is impacted as follows

$$
(\mathbf{M}+\tilde{\mathbf{M}}) \ddot{\mathbf{q}}+(\mathbf{G}+\tilde{\mathbf{G}}) \dot{\mathbf{q}}+(\mathbf{K}+\tilde{\mathbf{K}}) \mathbf{q}=\mathbf{0}
$$

Tilde matrices denote random contributions with zero mean. They depend on a set of random parameters denoted $\xi$. Reasoning in the same way as in the deterministic study, the following augmented formulation is used for the eigenvalue problem

$$
(\mathbf{A}+\tilde{\mathbf{A}}) \tilde{\mathbf{u}}_{k}=\tilde{\lambda}_{k}(\mathbf{B}+\tilde{\mathbf{B}}) \tilde{\mathbf{u}}_{k}
$$

with normalization equation

$$
{\overline{\tilde{\mathbf{u}}_{k}}}^{\mathrm{T}}(\mathbf{B}+\tilde{\mathbf{B}}) \tilde{\mathbf{u}}_{k}=1
$$

$\tilde{\mathbf{u}}_{k}$ and $\tilde{\lambda}_{k}$ respectively denote stochastic eigenvectors and eigenvalues.

Polynomial Chaos expansion consists in decomposing uncertain quantities on a basis of Hermite multivariate polynomials [6]. These polynomials are best suited for Gaussian stochastic processes. Non-Gaussian processes are classically decomposed on Gaussian processes [19]. The computation of the polynomials as well as the appropriate scalar product are recalled in Appendix A.

Matrices introducing uncertainty are then decomposed as follows, using a Karhunen-Loève decomposition or assuming them so regarding experimental results [6]:

$$
\tilde{\mathbf{X}}=\sum_{n=2}^{N_{X}} \mathbf{X}_{n} \boldsymbol{\psi}_{n}(\xi)
$$

where $\mathbf{X}$ can denote $\mathbf{M}, \mathbf{G}$ or $\mathbf{K} . \boldsymbol{\psi}_{1}$ will conventionally denote the constant polynomial equal to 1 (associated with deterministic component); polynomials with index greater or equal to 2 are zero mean.

In the proposed method, stochastic eigenvalues and eigenvectors of the augmented system in Eq. (6) with constraint (Eq. (7)) are decomposed on both the deterministic eigenmodes and the PC:

$$
\begin{gathered}
\tilde{\lambda}_{k}=\lambda_{k} \sum_{s=1}^{N}\left({ }^{(k)} a_{s}+\mathbf{j}^{(k)} b_{s}\right) \boldsymbol{\psi}_{s}(\xi) \\
\tilde{\mathbf{u}}_{k}=\sum_{p=1}^{P}\left({ }^{(k)} \tilde{\gamma}_{p}(\xi)+\mathbf{j}^{(k)} \tilde{\mu}_{p}(\xi)\right) \mathbf{u}_{p}
\end{gathered}
$$

with

$$
{ }^{(k)} \tilde{\gamma}_{p}(\xi)=\sum_{n=1}^{N}{ }^{(k)} \gamma_{p}^{n} \psi_{n}(\xi) \quad \text { and } \quad{ }^{(k)} \tilde{\mu}_{p}(\xi)=\sum_{n=1}^{N}{ }^{(k)} \mu_{p}^{n} \psi_{n}(\xi)
$$

giving

$$
\left.\tilde{\mathbf{u}}_{k}=\sum_{p=1}^{p} \sum_{n=1}^{N}\left({ }^{(k)} \gamma_{p}^{n}+\mathbf{j}^{(k)} \mu_{p}^{n}\right) \boldsymbol{\psi}_{n}(\xi)\right) \mathbf{u}_{p}
$$

where the coefficients ${ }^{(k)} \gamma_{p}^{n},{ }^{(k)} \mu_{p}^{n},{ }^{(k)} a_{s}$ and ${ }^{(k)} b_{s}$ are real and $\mathbf{j}$ denotes the imaginary unit $\left(\mathbf{j}^{2}=-1\right)$. This decomposition on $P$ deterministic eigenvalues and eigenvectors is a generalization of what is proposed in Ref. [16]. It lets real and imaginary parts of stochastic eigenvalues and eigenvectors evolve independently. In this paper, $P=2 n$, that is all the deterministic modes are used for projection of stochastic eigenvectors but a proper selection of the deterministic modes retained may be of great interest (see Sec. 3.3).

To get the final system of equations that leads to the unknowns ${ }^{(k)} \gamma_{p}^{n},{ }^{(k)} \mu_{p}^{n},{ }^{(k)} a_{s}$ and ${ }^{(k)} b_{s}$, Eqs. (6) and (7) are projected onto the Polynomial Chaos basis $\psi_{k}(\xi), 1 \leq k \leq N$ using the dedicated 
scalar product (Eq. (A7)). Real and imaginary parts of the subsequent equations are separated. This generates $2 P(P+1) N$ nonlinear (quadratic) equations, that is as many as unknowns. They can be solved through a general nonlinear solver.

\section{Application to a Finite Element Rotor Model}

In this section the PC expansion approach will be applied to a finite element (FE) model of a rotor. First, the deterministic model is exposed and characterized. Then, the three uncertain configurations considered in this study and their handling are described. In a third part, the PC expansion method is validated through a comparison with MC simulations and the computation cost question is addressed. Finally, physical meanings of results are commented.

3.1 Model and Deterministic Modal Characterization. The considered rotor consists in a shaft modeled by seven Timoshenko beam finite elements (four dofs per node) [18,20,21]. It is supported by two linear undamped bearings; each one is modeled by two orthogonal springs. The rotor has three rigid discs. Elements are located as shown in Fig. 1.

Numerical characteristics of these elements are:

Shaft: external diameter $D=0.01 \mathrm{~m}$; Young modulus $E=2 \cdot 10^{11} \mathrm{~Pa}$; shear coefficient $G=7.7 \cdot 10^{10} \mathrm{~Pa}$; density $\rho=7800 \mathrm{~kg} \cdot \mathrm{m}^{-3}$; length $L=0.7 \mathrm{~m}$; it is divided into 7 elements with length $L_{e}=0.1 \mathrm{~m}$.

Discs: internal diameter $D_{i}=0.01 \mathrm{~m}$, density $\rho=7800 \mathrm{~kg} . \mathrm{m}^{-3}$ outer diameter $D_{e}=0.2 \mathrm{~m}$ for disc 1 and disc 2 and $D_{e}=0.15 \mathrm{~m}$ for disc 3.

Bearings: stiffness in each orthogonal direction $k=1 \cdot 10^{7}$ N.m ${ }^{-1}$.

Equations of the system are written in the fixed frame. Contributions of each element are detailed below and detailed matrices expressions can be found in Appendix B.

The equations of motion of the shaft can be written as:

$$
\left(\mathbf{M}_{s T}+\mathbf{M}_{s R}\right) \ddot{\mathbf{q}}_{s}+\mathbf{G}_{s} \dot{\mathbf{q}}_{s}+\mathbf{K}_{s} \mathbf{q}_{s}=\mathbf{0}
$$

where $\mathbf{q}_{s}$ defines the vector of displacements and rotations of the shaft. $\mathbf{M}_{s T}$ and $\mathbf{M}_{s R}$ are the translational and rotary mass matrices. $\mathbf{G}_{s}$ is the gyroscopic matrix relative to the shaft, skew-symmetric and proportional to the rotation speed $\Omega$ and $\mathbf{K}_{s}$ is the stiffness matrix. Each matrix size is $32 \times 32$.

Each rigid disc $n(1 \leq n \leq 3)$ contributes in the following way:

$$
\left(\mathbf{M}_{d n T}+\mathbf{M}_{d n R}\right) \ddot{\mathbf{q}}_{d b}+\mathbf{G}_{d n} \dot{\mathbf{q}}_{d n}=\mathbf{0}
$$

where $\mathbf{q}_{d n}$ defines the displacements and rotations vector of disc $n$. $\mathbf{M}_{d n T}$ and $\mathbf{M}_{d n R}$ are the translational and rotary mass matrices. $\mathbf{G}_{d n}$ is the gyroscopic matrix, skew-symmetric and proportional to the rotation speed. Each matrix has size $4 \times 4$. Finally, each bearing is represented by a stiffness matrix $\mathbf{K}_{b n}$ (with $1 \leq n \leq 2$ ) of size $2 \times 2$.

Assembling the matrices of the different contributions (i.e. the shaft, the two bearings and the three discs), one gets the complete equation of motion of the rotor system in the case of free vibrations:

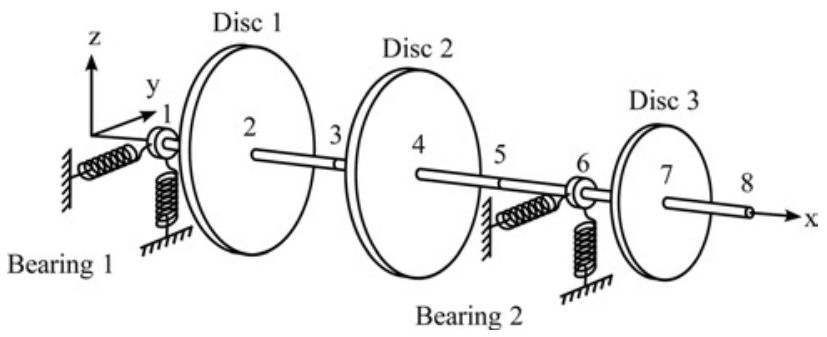

Fig. 1 FE rotor: plan and node numbers

$$
\mathbf{M} \ddot{\mathbf{q}}+\mathbf{G} \dot{\mathbf{q}}+\mathbf{K q}=\mathbf{0}
$$

where $\mathbf{q}$ defines the vector of displacements and rotations of the complete rotor system. $\mathbf{M}$ and $\mathbf{K}$ are, respectively, mass and stiffness matrices. Mass matrix includes contributions of the shaft and the discs while stiffness matrix includes contributions of the shaft and the bearings. These matrices are symmetric. $\mathbf{G}$ is the gyroscopic matrix, skew-symmetric and proportional to $\Omega$; it includes the contributions of the shaft elements and the three discs. As stated in the system description, no damping is taken into account neither in the shaft nor in the bearings. Total size of the system is $8 \times 4=32$.

Computing eigenvalues of this system for several rotation speeds (as mentioned in Sec. 2, Eq. (3)), the classical Campbell diagram is built and displayed in Fig. 2.

As depicted on this graphic, one will focus on the first three double modes (denoted $\mathrm{nF}$ for the $\mathrm{n}$-th forward whirl mode and $\mathrm{nB}$ for the related $n$-th backward whirl mode) for frequency range $[0,70]$ Hz. The line $\omega=\Omega$ is displayed to help the reading of critical speeds (when an eigenvalue equals the rotation speed). The first critical speeds in the deterministic case are then $\Omega_{c 1 B}=10.46 \mathrm{~Hz}$, $\Omega_{c 1 F}=11.98 \mathrm{~Hz}, \Omega_{c 2 B}=23.91 \mathrm{~Hz}, \Omega_{c 2 F}=34.12 \mathrm{~Hz}, \Omega_{c 3 B}=43.54 \mathrm{~Hz}$ and $\Omega_{c 3 F}=51.87 \mathrm{~Hz}$.

3.2 Uncertain Cases and Processing. In the following section of the paper, three uncertain configurations will be tested. In each case, uncertainties are introduced as independent real standard normal variables.

Case 1: uncertain Young modulus of the shaft. $E$ varies with a standard deviation equal to $6 \%$ of its mean value. This globally impacts the stiffness matrix (see elementary matrix Eq. (B3)) leading to $N_{K}=2.99 \%$ of the occurrences belong to range $\left[1.64 \cdot 10^{11}, 2.36 \cdot 10^{11}\right] \mathrm{Pa}$.

Case 2: uncertain density of first disc. $\rho$ varies according to a Gaussian with standard deviation equal to $10 \%$ of its initial value. This uncertainty implies a variation of both mass and gyroscopic matrices (see Appendix Eqs. (B5), (B6) and (B7)): $N_{M}=2$ and $N_{G}=2$

Case 3: both shaft Young modulus and first disc density independently with respective standard deviations equal to $4 \%$ and $3 \%$ of their mean value. This impacts the three mass, gyroscopic and stiffness matrices. In this case, $99 \%$ of Young modulus values rely in $\left[1.76 \cdot 10^{11}, 2.24 \cdot 10^{11}\right] \mathrm{Pa}$ and $99 \%$ of first disc density values are in range $[7098,8502] \mathrm{kg} \cdot \mathrm{m}^{-3}$.

The first two cases will emphasize the ability of the proposed method to process large dispersion ranges while the third one reflects more realistic ranges and will test its robustness and efficiency when dealing with multiple uncertain parameters.

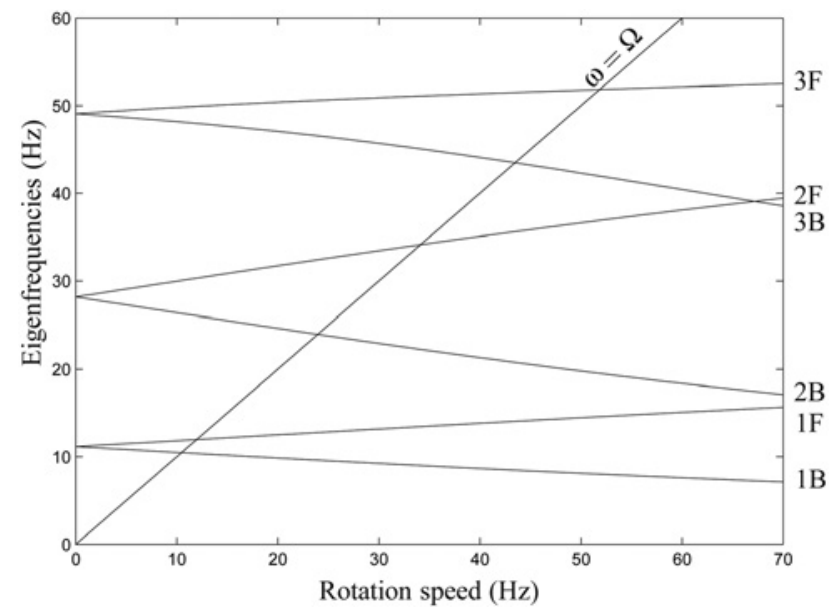

Fig. 2 FE rotor: deterministic Campbell diagram 


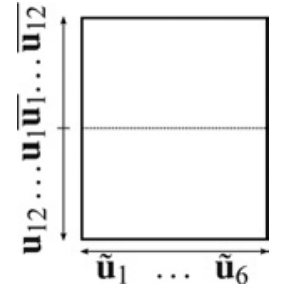

Fig. 3 MAC-like plot arrangement

In order to rebuild the Campbell diagram in the different uncertain cases, eigenvalues and eigenvectors are evaluated for fifteen rotation speeds, namely $\Omega \in[0,5,10, \ldots, 65,70] \mathrm{Hz}$ : first, the six eigenmodes PC expansions are computed as exposed in Sec. 2. Then, 10,000 occurrences are evaluated on the same sample of $\xi$ points, using both the projections and a reference Monte Carlo simulation.

To deal with this great amount of data, an adaptation of the MAC (Modal Accordance Criterion) will be exposed for analysis of results on eigenvectors. Moreover, a way to evaluate critical speeds dispersion and corresponding eigenvectors evolution without having to do further projections will be proposed. These two points are detailed below.

3.2.1 Eigenvectors Dispersion. The Modal Accordance Criterion (MAC) and similar criteria [22] are usual tools for comparing experimental and numerical results. As stochastic modes are decomposed along deterministic ones, as stated in Eq. (10), a way to characterize their dispersion is to monitor $\left|{ }^{(k)} \tilde{\gamma}_{p}(\xi)+\mathbf{j}^{(k)} \tilde{\mu}_{p}(\xi)\right|$, that is the contribution of deterministic mode $p$ to stochastic mode $k$. This monitoring is close to the one obtained with a MAC using the scalar product defined by the mass matrix rather than the hermitian one. As polynomials $\psi_{n}$ are zero mean for $n \geq 2$, one has

$$
E\left[\left|{ }^{(k)} \tilde{\gamma}_{p}(\xi)+\mathbf{j}^{(k)} \tilde{\mu}_{p}(\xi)\right|\right]=\left|{ }^{(k)} \gamma_{p}^{1}+\mathbf{j}^{(k)} \mu_{p}^{1}\right|
$$

However, the standard deviation cannot be obtained readily because of the modulus embracing the complex coefficients. Thus it is rebuilt using evaluation of the coefficients on a large sample (10,000 occurrences). Results are presented as for a MAC, by displaying mean and standard deviation of deterministic modes contributions for each stochastic mode studied.

Stochastic modes are placed along abscissa while contributions of some deterministic modes are plotted along y axis. As considering every deterministic mode may not be relevant (high frequency modes do not mix with low frequency ones), the resulting graphics will focus on the contributions of the first twelve deter-

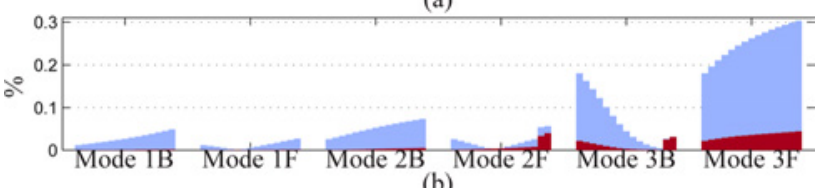

(b)

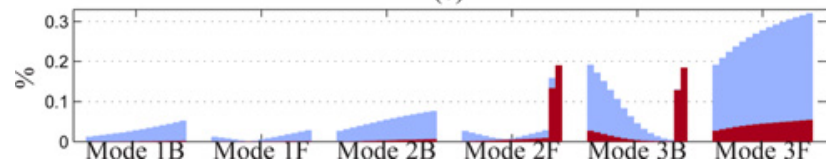

Fig. 5 Case 2: relative error on eigenvalues (\%); (a) mean and (b) standard deviation. Light blue, order 1 PC and dark red, order $\mathbf{2}$ PC. Each group of values is relative to a mode. For one mode, each bar represents a different speed $\Omega=0,5, \ldots, 70 \mathrm{~Hz}$. (Please check the online version for color figures)

ministic modes and their complex conjugates which are also eigenmodes. These quantities will be ordered as shown by Fig. 3 .

3.2.2 Critical Speeds. To find the critical speed for mode $n$, one has to solve $\omega_{n}(\Omega)=\Omega$. As the evolution of the eigenvalues with $\Omega$ is almost linear for these first modes, it seems fair enough to interpolate $\omega_{n}(\Omega)$ by using a quadratic polynomial based on three known values. In this study, it has been chosen to interpolate the eigenvalues using rotation speeds $\Omega=5 \mathrm{~Hz}, 35 \mathrm{~Hz}$ and $65 \mathrm{~Hz}$. For each occurrence, and for the $n$-th eigenvalue, an order 2 polynomial $\omega_{n}^{\text {int }}(\Omega)$ approximates $\omega_{n}(\Omega)$. It is then easy to solve $\omega_{n}^{\text {int }}(\Omega)=\Omega$ which has two solutions, the relevant one being the smallest here. Finally, for each uncertain case, one gets 10,000 sets of the first six critical speeds.

To get the corresponding eigenvectors, a similar interpolation is done on each component of each eigenvector for the same rotation speeds $\Omega$ (i.e. $5 \mathrm{~Hz}, 35 \mathrm{~Hz}$ and $65 \mathrm{~Hz}$ ). The approximate eigenvectors are then evaluated at the corresponding critical speeds (previously determined). This lets us draw the dispersion on eigenshapes at critical speeds.

3.3 Approximation Performance. This section is devoted to the validation of the method by comparing the results obtained using PC expansions of order 1 and 2 with Monte Carlo simulations in terms of error quantification and time consumption.

3.3.1 PC Approximation Error. For all the results of the three uncertain cases studied, both eigenvalues and eigenvectors are examined. For eigenvalues, the relative error on imaginary part $\omega$ histogram is computed for each mode and each rotation speed. The absolute relative error formula used is:

(a)

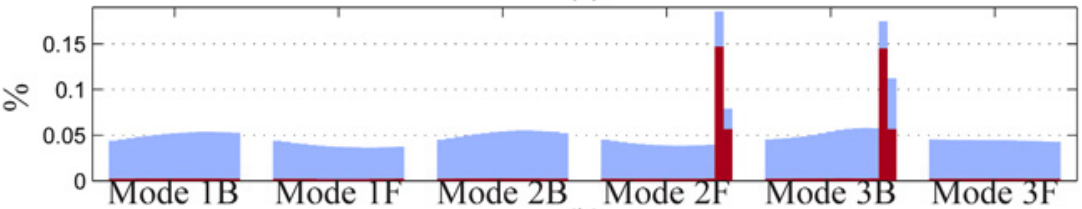

(b)

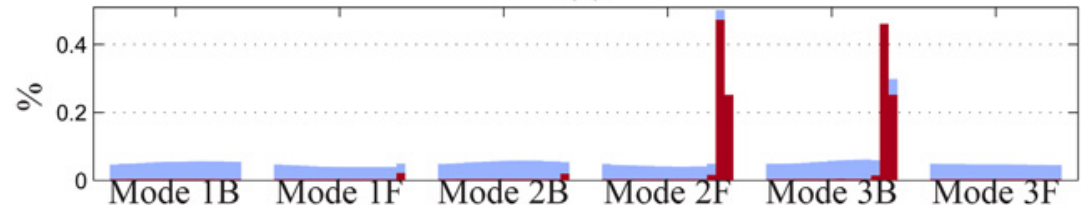

Fig. 4 Case 1: relative error on eigenvalues (\%); (a) mean and (b) standard deviation; light blue, order 1 PC and dark red, order 2 PC. Each group of values is relative to a mode. For one mode, each bar represents a different speed $\Omega=0,5, \ldots, 70 \mathrm{~Hz}$. (Please check the online version for color figures) 
(a)

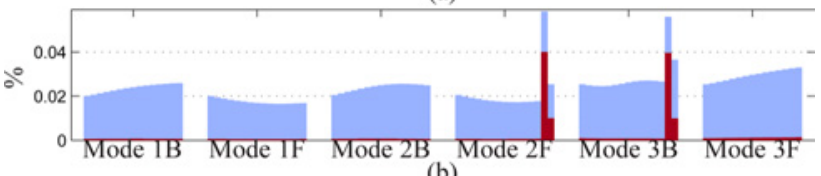

(b)

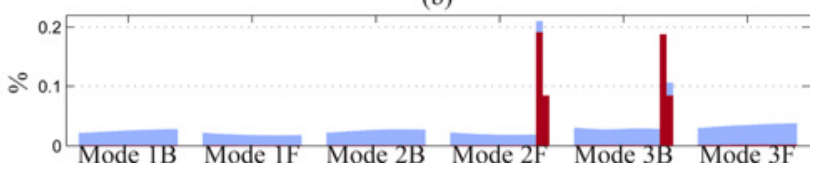

Fig. 6 Case 3: relative error on eigenvalues (\%); (a) mean and (b) standard deviation. Light blue, order 1 PC and dark red, order 2 PC. Each group of values is relative to a mode. For one mode, each bar represents a different speed $\Omega=0,5, \ldots, 70 \mathrm{~Hz}$. (Please check the online version for color figures)

(a)

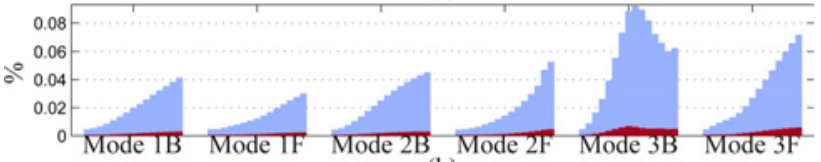

(b)

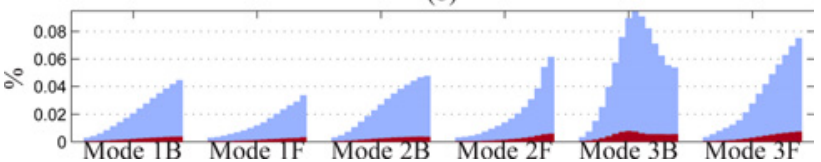

Fig. 7 Case 1: relative error on eigenvectors (\%); (a) mean and (b) standard deviation. Light blue, order 1 PC and dark red, order 2 PC. Each group of values is relative to a mode. For one mode, each bar represents a different speed $\Omega=0,5, \ldots, 70 \mathrm{~Hz}$. (Please check the online version for color figures)

(a)

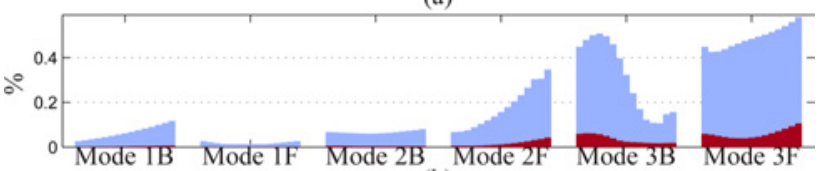

(b)

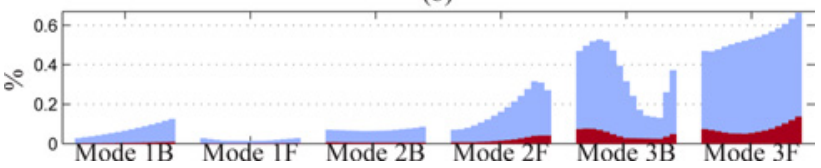

Fig. 8 Case 2: relative error on eigenvectors (\%); (a) mean and (b) standard deviation. Light blue, order 1 PC and dark red, order 2 PC. Each group of values is relative to a mode. For one mode, each bar represents a different speed $\Omega=0,5, \ldots, 70 \mathrm{~Hz}$. (Please check the online version for color figures)

(a)
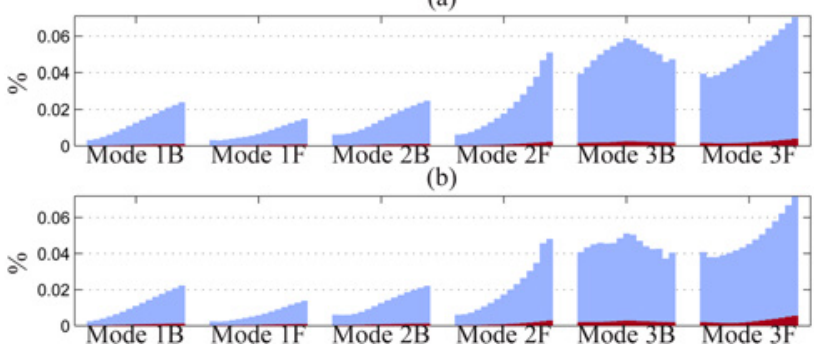

Fig. 9 Case 3: relative error on eigenvectors (\%); (a) mean and (b) standard deviation; light blue, order $1 \mathrm{PC}$ and dark red, order 2 PC. Each group of values is relative to a mode. For one mode, each bar represents a different speed $\Omega=0,5, \ldots, 70 \mathrm{~Hz}$. (Please check the online version for color figures)
Table 1 Computation times (cpu s) for Polynomial Chaos approximation of order 1 and 2 and Monte Carlo simulations (MC)

\begin{tabular}{lccr}
\hline \hline Case & $\begin{array}{c}\text { Order 1 PC } \\
t_{\text {fit }}+t_{\text {shoot }}=t_{\text {tot }}\end{array}$ & $\begin{array}{c}\text { Order 2 PC } \\
t_{\text {fit }}+t_{\text {shoot }}=t_{\text {tot }}\end{array}$ & MC \\
\hline 1 & $5+3=8$ & $10+3=13$ & 467 \\
2 & $5+3=8$ & $12+3=15$ & 462 \\
3 & $13+3=16$ & $82+3=85$ & 476 \\
\hline
\end{tabular}

Table 2 Number of polynomials involved $(M)$ and system size for case 1 and case 2

\begin{tabular}{lcc}
\hline \hline & $\begin{array}{c}\text { Order 1 PC } \\
\text { approximation }\end{array}$ & $\begin{array}{c}\text { Order 2 PC } \\
\text { approximation }\end{array}$ \\
\hline$N$ & 2 & 3 \\
Syst. size & 260 & 390 \\
\hline \hline
\end{tabular}

(a.1)

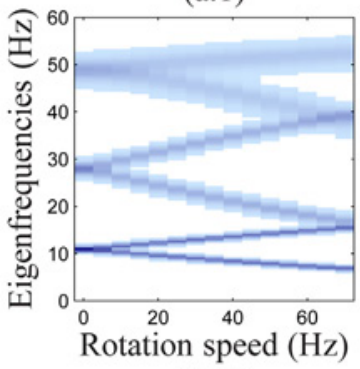

(b.1)

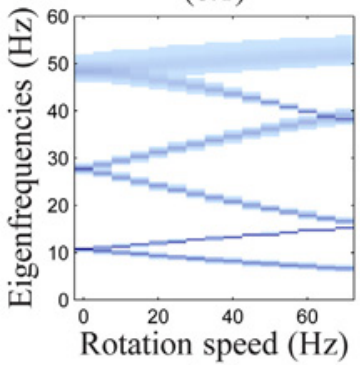

(c.1)

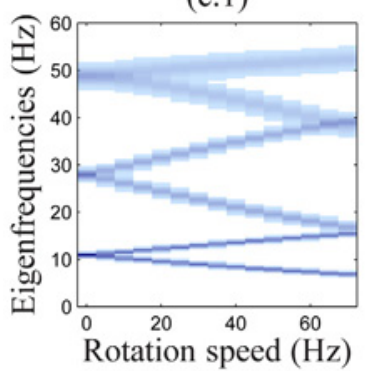

(a.2)

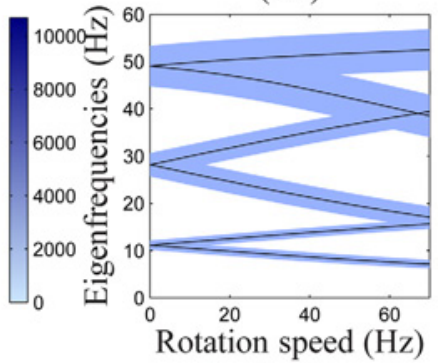

(b.2)

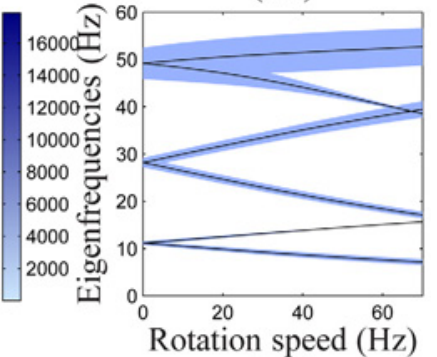

(c.2)

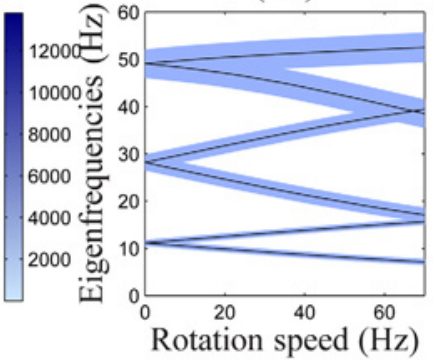

Fig. 10 Stochastic Campbell diagrams for (a) case 1, (b) case 2 and $(c)$ case 3 ; (x.1): histograms, colorbar indicates the number of occurrences; (x.2): mean value (-) and mean value $\pm 3 \times$ standard deviation (light blue patch) (Please check the online version for color figures)

$$
e_{\omega_{k, j}}=\left|\frac{\omega_{k, j}^{\mathrm{MC}}-\omega_{k, j}^{\mathrm{PC}}}{\omega_{k, j}^{\mathrm{MC}}}\right| \times 100
$$

where $\omega_{k, j}^{\mathrm{MC}}$ denotes the $\mathrm{k}$-th eigenvalue computed directly for the $\mathrm{j}$-th point $\xi_{j}$ while $\omega_{k, j}^{\mathrm{PC}}$ is the one evaluated using Eq. (9) for PC expansions of order 1 or order 2. 
(a)
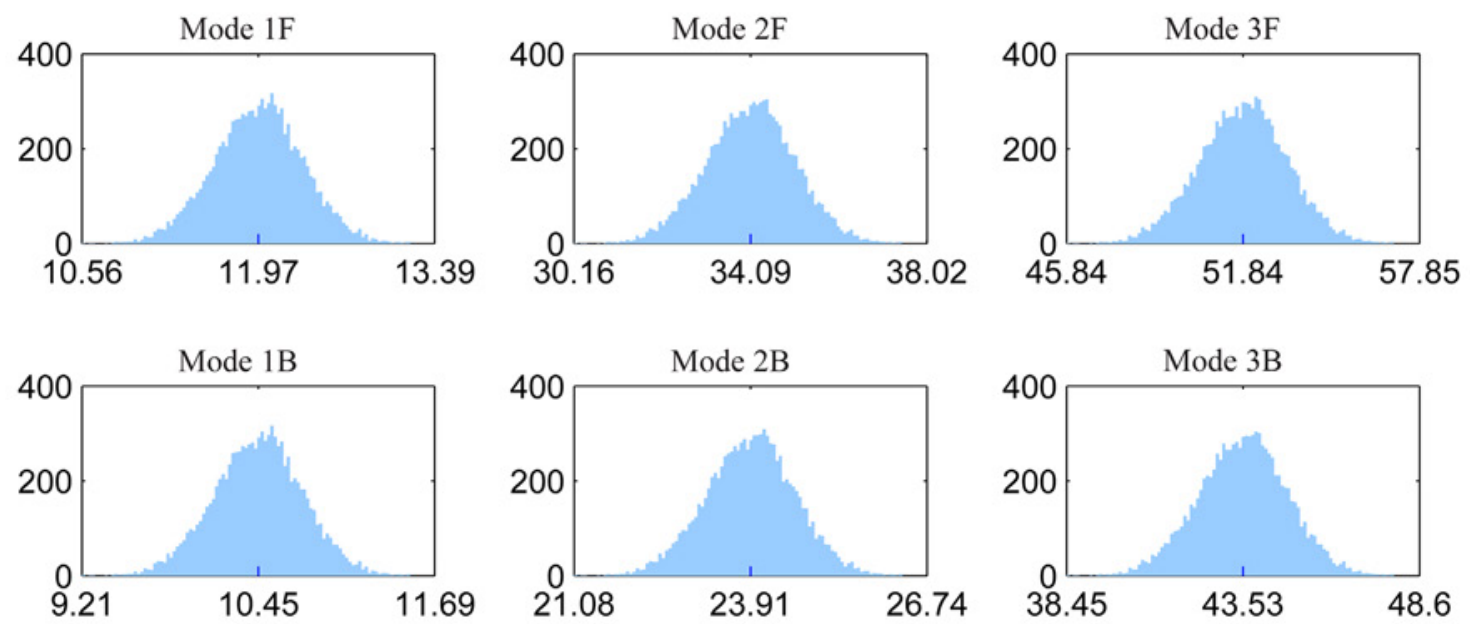

(b)
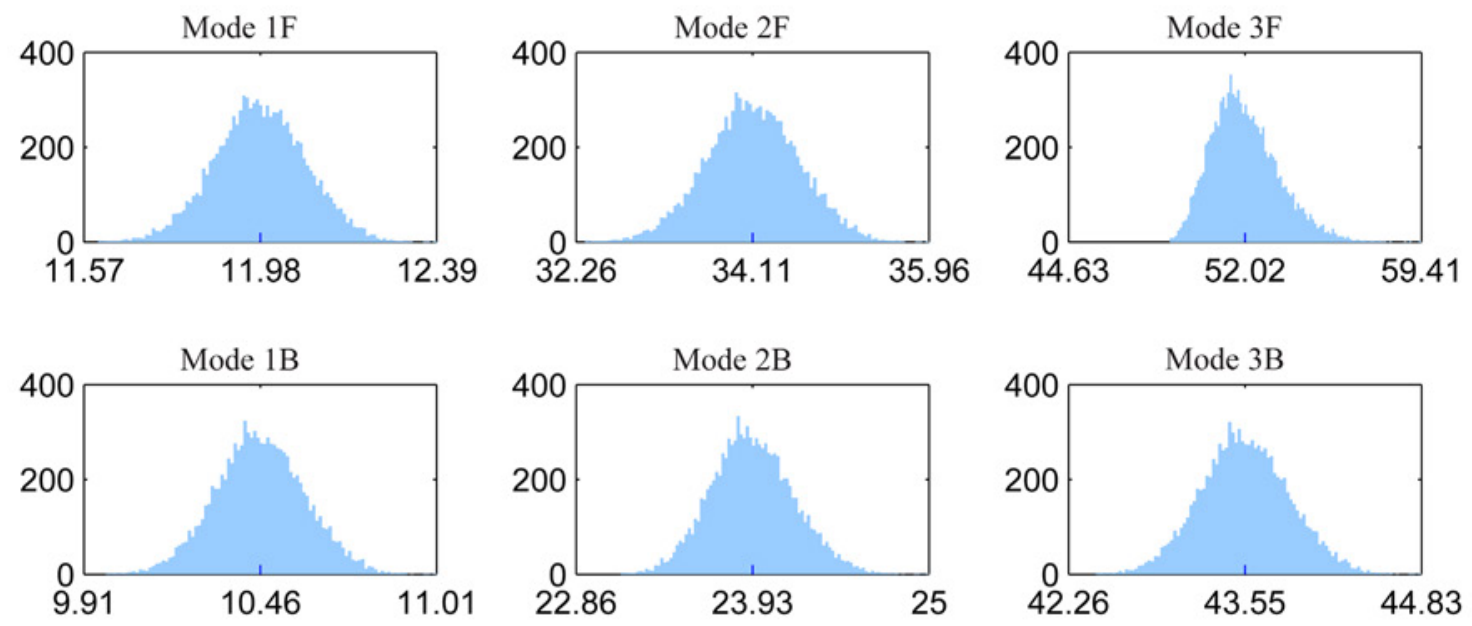

(c)
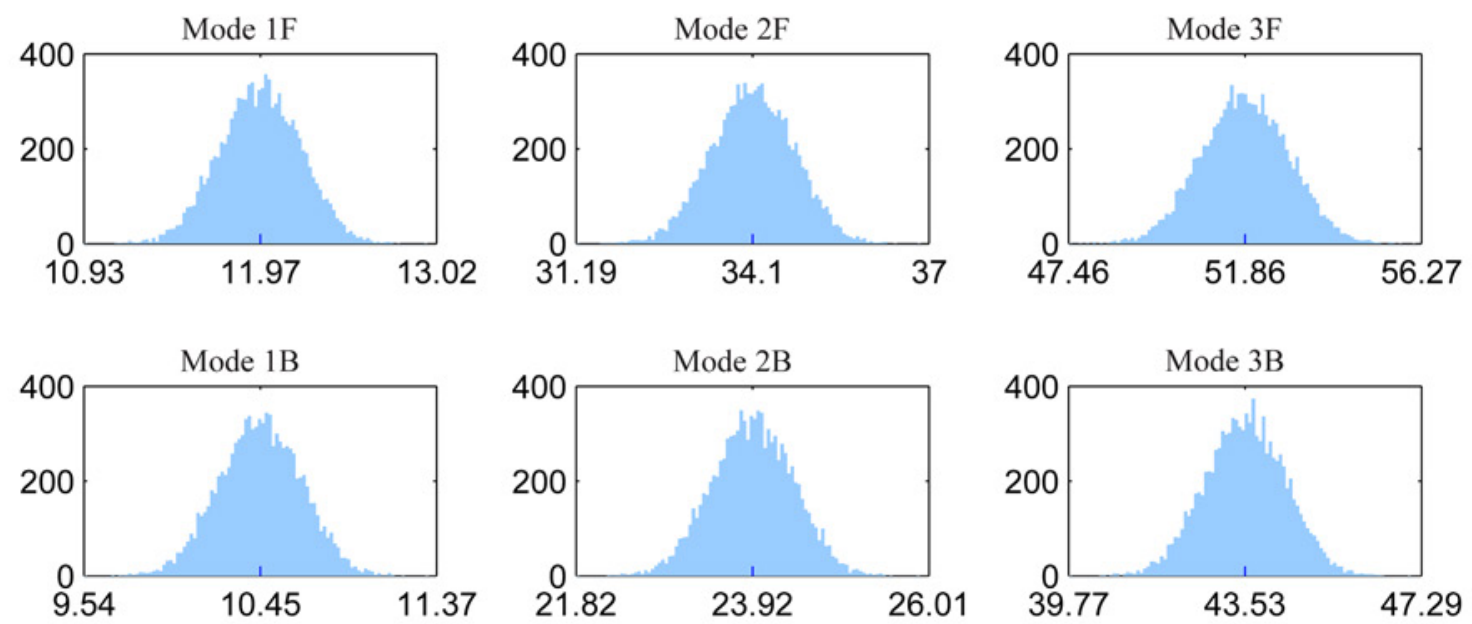

Fig. 11 Critical speeds dispersion: number of occurrences versus critical speed value $(\mathrm{Hz})$ for (a) case 1, (b) case 2 and $(c)$ case 3

Comparing eigenvectors is a little bit more difficult as they are not scalar quantities; one then has to define a way to sum up the information, and as they may not be all in phase: given no excita- tion, pairs of backward and forward modes eigenvectors are orthogonal but they may define different orthogonal planes for $t=0$ for example. To overcome this problem, for each mode 
(a)
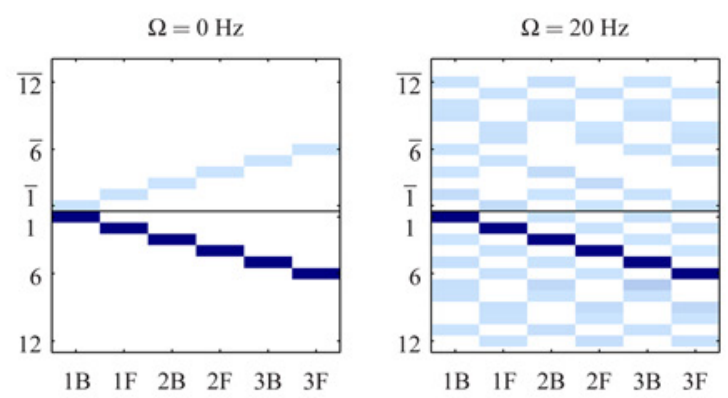

$\begin{array}{llllll}1 \mathrm{~B} & 1 \mathrm{~F} & 2 \mathrm{~B} & 2 \mathrm{~F} & 3 \mathrm{~B} & 3 \mathrm{~F}\end{array}$

$\Omega=45 \mathrm{~Hz}$

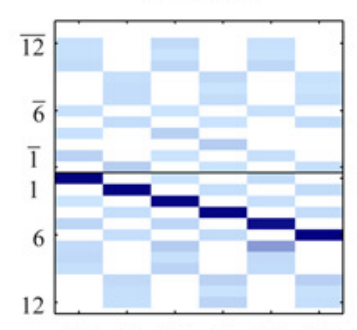

$\begin{array}{llllll}1 \mathrm{~B} & 1 \mathrm{~F} & 2 \mathrm{~B} & 2 \mathrm{~F} & 3 \mathrm{~B} & 3 \mathrm{~F}\end{array}$

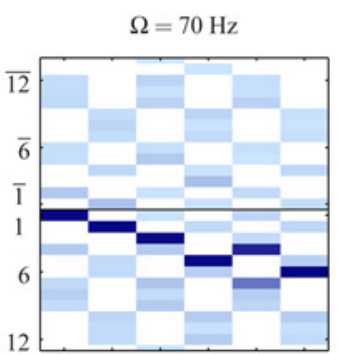

$\begin{array}{llllll}1 \mathrm{~B} & 1 \mathrm{~F} & 2 \mathrm{~B} & 2 \mathrm{~F} & 3 \mathrm{~B} & 3 \mathrm{~F}\end{array}$

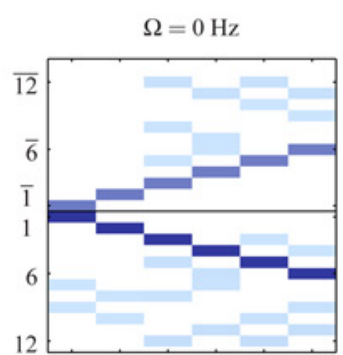

$\begin{array}{llllll}1 \mathrm{~B} & \text { IF } & 2 \mathrm{~B} & 2 \mathrm{~F} & 3 \mathrm{~B} & 3 \mathrm{~F}\end{array}$

(b)

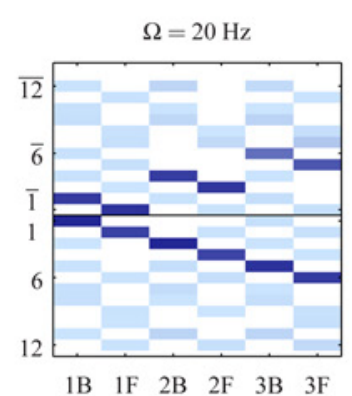

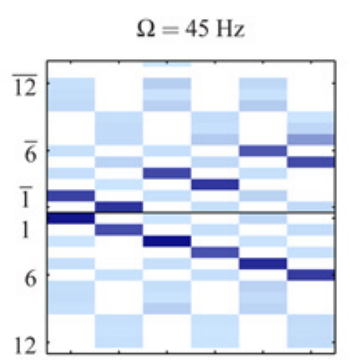

$\begin{array}{llllll}1 \mathrm{~B} & 1 \mathrm{~F} & 2 \mathrm{~B} & 2 \mathrm{~F} & 3 \mathrm{~B} & 3 \mathrm{~F}\end{array}$

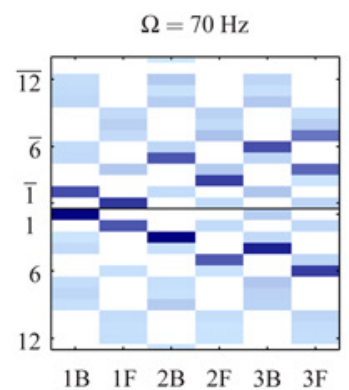

IB $\quad 1 \mathrm{~F} \quad 2 \mathrm{~B} \quad 2 \mathrm{~F} \quad 3 \mathrm{~B} \quad 3 \mathrm{~F}$
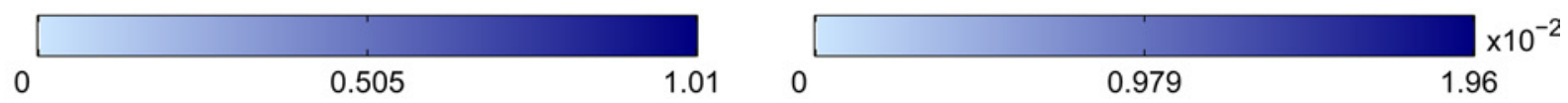

Fig. 12 Eigenvectors dispersion: (a) mean and (b) standard deviation of stochastic weights of deterministic modes 1 to 12 and conjugates for PC expansion of order 2 for uncertain case 1

occurrence $\mathbf{u}_{k, j}^{\mathrm{PC}}\left(k\right.$-th mode for $j$-th realization set $\xi_{j}$ using PC formula Eq. (12)), the component $l$ with maximum amplitude is used to computed an angle $\theta$ :

$$
\theta=\tan ^{-1}\left(\frac{\operatorname{Im}\left(u_{k, j, l}^{\mathrm{PC}}\right)}{\operatorname{Re}\left(u_{k, j, l}^{\mathrm{PC}}\right)}\right)
$$

This angle is used to rotate $\mathbf{u}_{k, j}^{\mathrm{PC}}$ by multiplying it by $\cos (-\theta)+\mathbf{j}$ $\sin (-\theta)$. Then the same $l$ component is used to compute the angle $\theta^{\prime}$ for the equivalent eigenvector obtained during the Monte Carlo simulation $\mathbf{u}_{k, j}^{\mathrm{MC}}$. This eigenvector is also rotated with $-\theta^{\prime}$ angle in the complex plane. Finally components relative to translational dofs, $l \in \mathcal{T}$, and rotational dofs, $l \in \mathcal{R}$, are separated to get the following absolute relative error for the $j$-th realization of mode $k$ :

$e_{u_{k, j}}=\max \left(\frac{\max _{l \in \mathcal{T}}\left(\left|u_{k, j, l}^{\mathrm{PC}}-u_{k, j, l}^{\mathrm{MC}}\right|\right)}{\max _{l \in \mathcal{T}}\left(\left|u_{k, j, l}^{\mathrm{MC}}\right|\right)}, \frac{\max _{l \in \mathcal{R}}\left(\left|u_{k, j, l}^{\mathrm{PC}}-u_{k, j, l}^{\mathrm{MC}}\right|\right)}{\max _{l \in \mathcal{R}}\left(\left|u_{k, j, l}^{\mathrm{MC}}\right|\right)}\right)$

$$
\times 100
$$

Histograms representing the mean value and the standard deviation of the error per mode and for each rotating speed can then be built.

Indicators relative to eigenvalues are plotted on Figs. 4, 5 and 6. For each mode, the 15 rotation speeds (i.e. $\Omega=0,5, \ldots$, $70 \mathrm{~Hz}$ ) are used to draw a bar of the histogram representing the mean or the standard deviation of the absolute relative error. As one can see, the error is negligible (far less than 1\%) and PC expansion of order 2 generally gives better results than order $1 \mathrm{PC}$ expansion.

As the proposed method lets real part of eigenvalues evolve independently from the imaginary part, it has been verified that for the studied examples the fitted real part were negligible (less than $\left.1 \cdot 10^{-12}\right)$.

Figures 7 and 8 and Fig. 9 display results relative to eigenvectors. These plots demonstrate a very good accordance between Monte Carlo simulations and PC expansions with a better performance observed for order $2 \mathrm{PC}$ expansion than for order $1 \mathrm{PC}$ expansion.

Globally, Monte Carlo simulations and Polynomial Chaos based results are in very good agreement for the three processed cases which lets us conclude that the method returns very accurate results.

3.3.2 Time Consumption. The main interest of the methodology using the Polynomial Chaos expansion is to be faster than the Monte Carlo simulations. Computational durations are given in Table 1. Computations were performed on an Intel Q6700 ( $3 \mathrm{GHz}$ ) with 8 Go of RAM; being a 4 core and most of calculus taking advantage of it, real time is approximately a fourth of the cpu times given in the table. Durations relative to PC approximation correspond to the computation of the first six modes. The sizes of systems solved for each mode are given in Table 2. As can be seen in these tables, getting the PC approximation coefficients is the most consuming step of the method (variable $t_{\text {fit }}$ in Table 1) while evaluating the 10,000 occurrences is really quick (variable $t_{\text {shoot }}$ in Table 1). Then, it is obvious that PC coefficient evaluation is advantageous when a great number of occurrences has to be computed. This approach also allows the test of different inputs as soon as they respect the decomposition form (Eq. (8)) for which PC coefficients have been computed once for all: different configurations can then be tested with more or less dispersion on input variables corresponding to a lesser or greater improvement and cost on the fabrication stage for example. Moreover, the fitting cost can be heavily decreased by selecting a set of deterministic modes instead of all; pertinence of this selection will be addressed in Sec. 3.4. Finally it is worth noting that the proposed method allows fitting for the $k$-th mode only, $k$ being as great as wanted, limited only by the refinement of the model. 
(a)
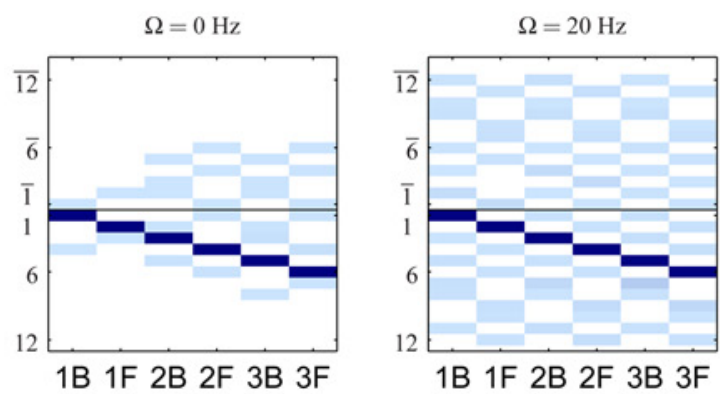

$\Omega=45 \mathrm{~Hz}$

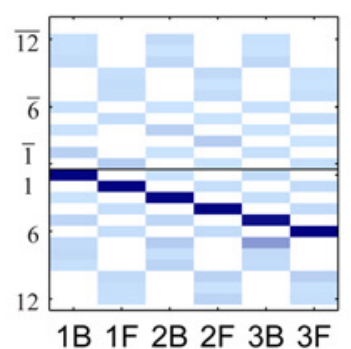

$\Omega=70 \mathrm{~Hz}$

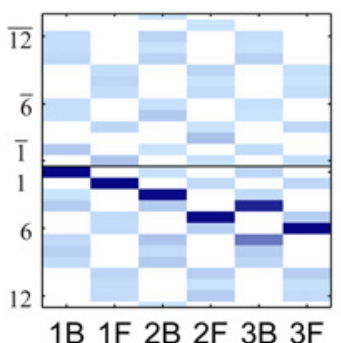

$\Omega=0 \mathrm{~Hz}$

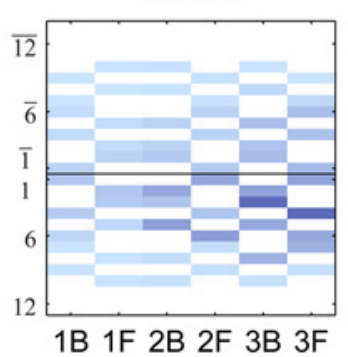

(b)
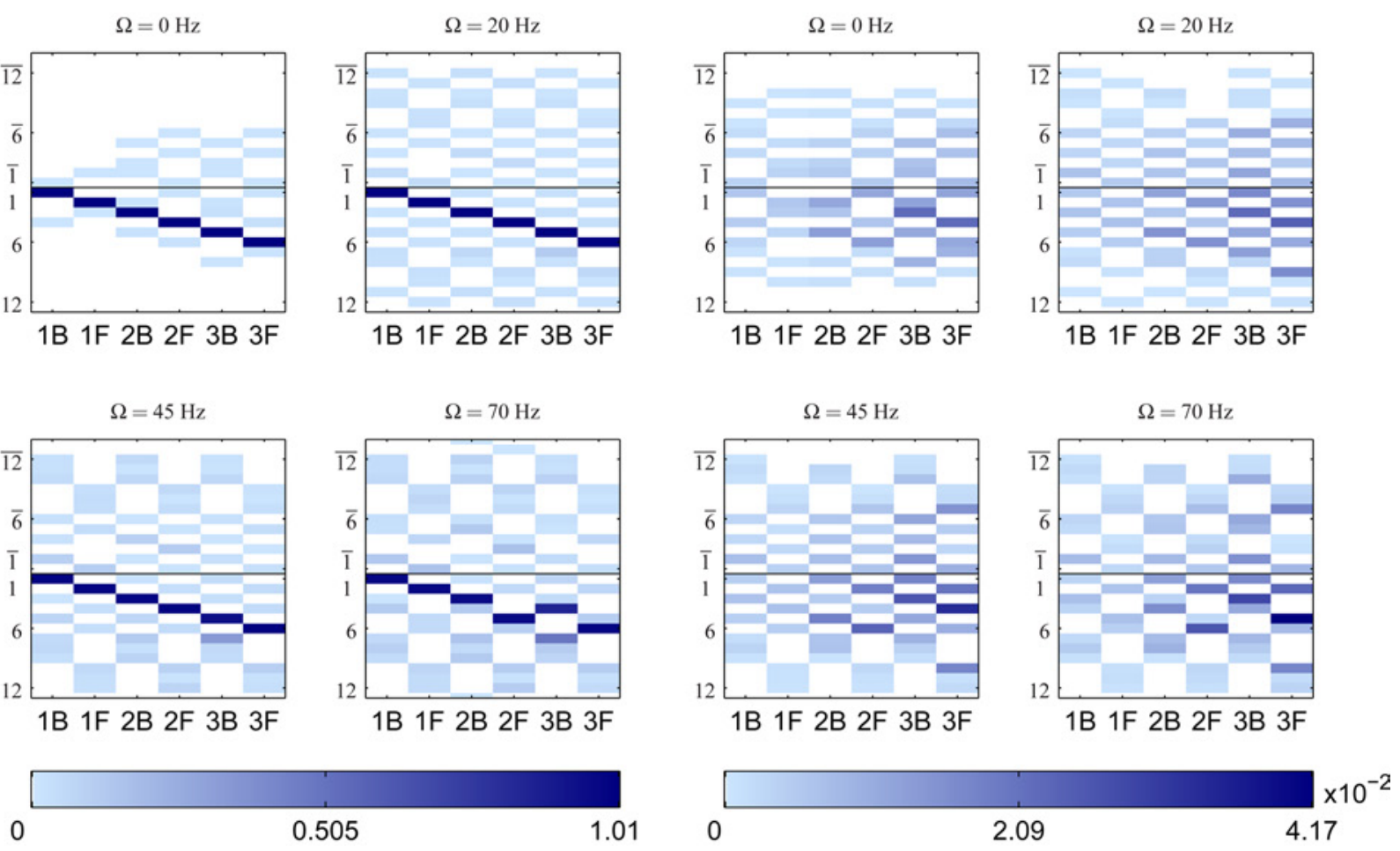

Fig. 13 Eigenvectors dispersion: (a) mean and $(b)$ standard deviation of stochastic weights of deterministic modes 1 to 12 and conjugates for PC expansion of order 2 for uncertain case 2

3.4 Physical Comments on Results. First, results on eigenvalues are exposed: stochastic Campbell diagrams and critical speeds dispersion are drawn and commented. In a second time, impact of uncertainty on eigenvectors is discussed using the MAC type indicator to monitor its evolution with rotation speed and the drawing of standard deviations of deformed shapes at critical speeds. (a)

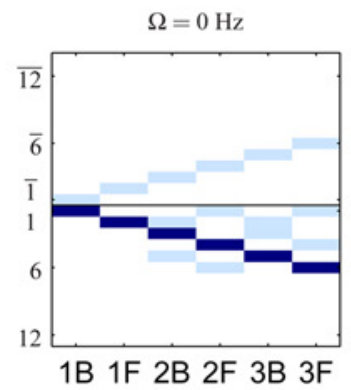

$\Omega=45 \mathrm{~Hz}$

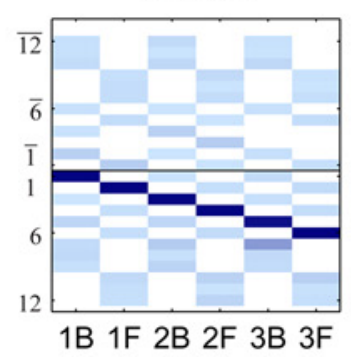

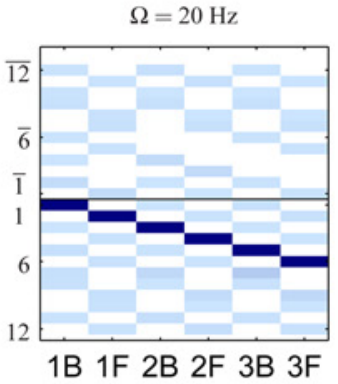

$\Omega=70 \mathrm{~Hz}$

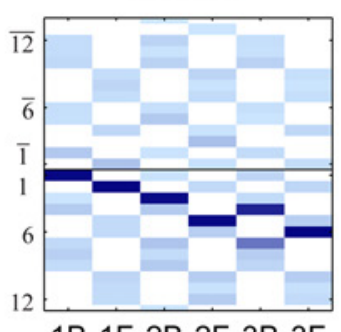

1B 1F 2B 2F 3B 3F

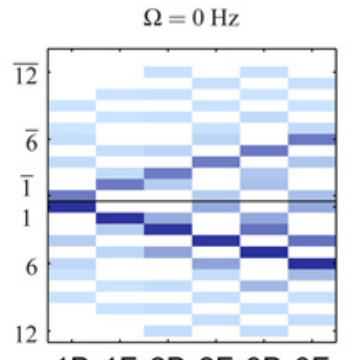

1B 1F 2B 2F 3B 3F (b)

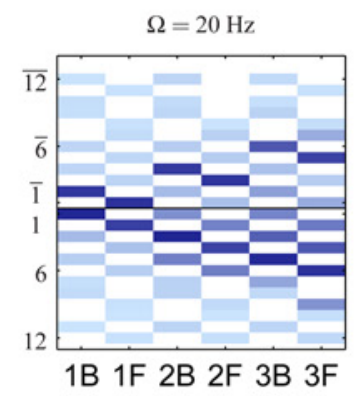

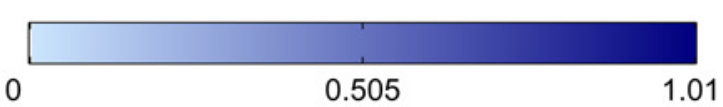

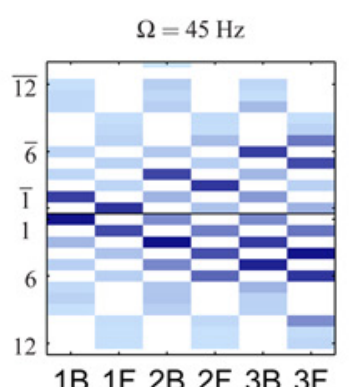

1B 1F 2B 2F 3B 3F

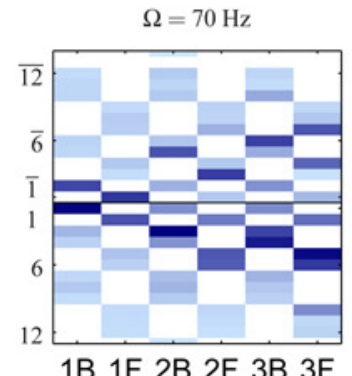

1B 1F 2B 2F 3B 3F

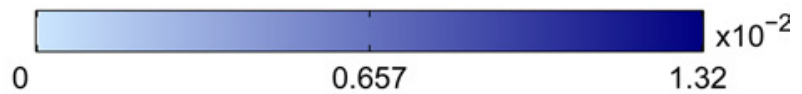

Fig. 14 Eigenvectors dispersion: (a) mean and (b) standard deviation of stochastic weights of deterministic modes 1 to 12 and conjugates for PC expansion of order 2 for uncertain case 3 
(a)

Mode $1 \mathrm{~F}$

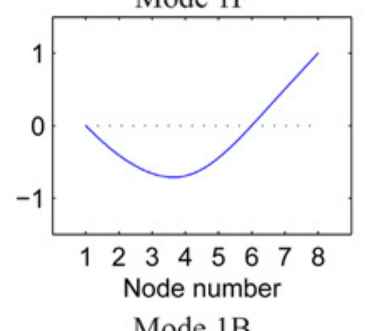

Mode 1B

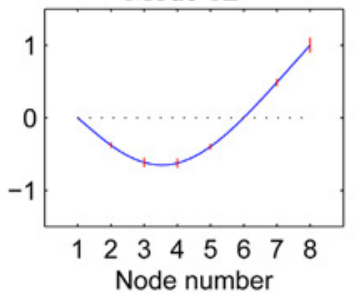

(b)

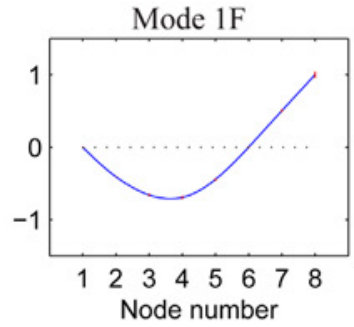

Mode 1B

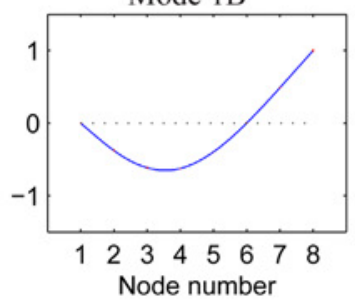

(c)

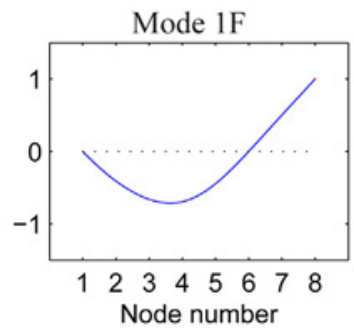

Mode 1B

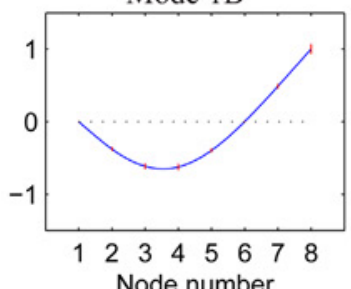

Mode 2F

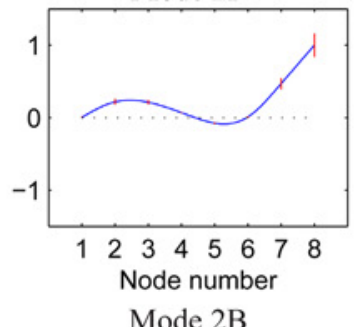

Mode 2B

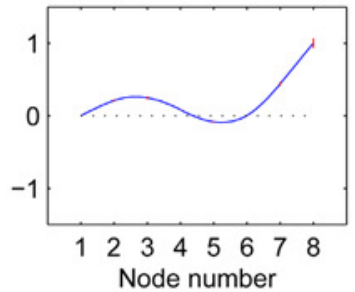

Mode 2F

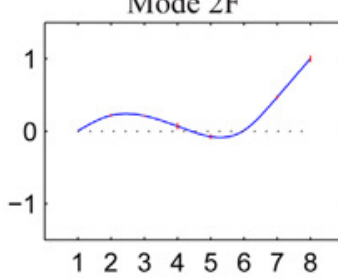

Node number

Mode 2B

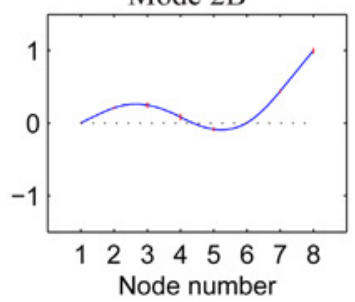

Mode 2F

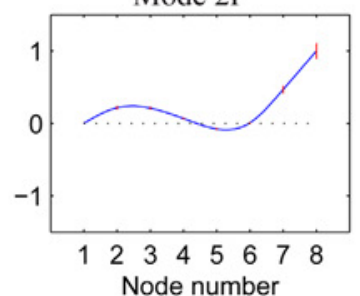

Mode 2B

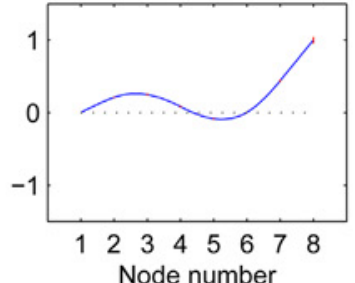

Mode 3F

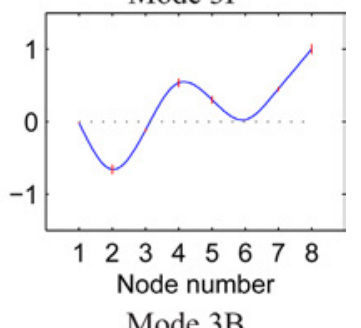

Mode 3B

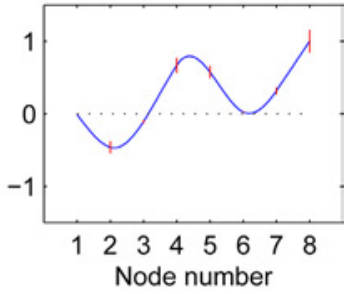

Mode 3F

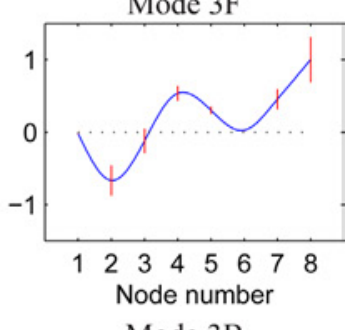

Mode 3B

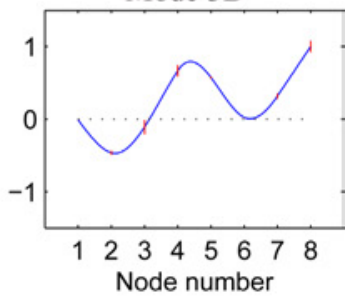

Fig. 15 Critical modeshapes dispersion: - mean shape (interpolated), I mean $\pm 3 \times$ standard deviation at nodes; (a) case $1,(b)$ case 2 and (c) case 3

3.4.1 Eigenvalues Dispersion. Figure 10 exposes the stochastic Campbell diagrams for the three uncertain cases. Left panes (set 1 of Fig. 10(a), 10(b), 10(c)) represent histograms giving a precise information about eigenvalues repartition while right panes (set 2 of Fig. 10(a), 10(b), 10(c)) give a more synthetic view by displaying the mean value for each mode (black line) and 
a blue patch covering the mean plus or minus three times the standard deviation which gives a good approximation of the location of most of the occurrences.

Dispersion is important and constant with rotation speed in case 1 both for eigenvalues (set 1 of Fig. 10(a) and set 2 of 10(a)) and critical speeds (Fig. 11(a)). This can be explained by very small variations of mode shapes with rotation speed. As uncertainty affects shaft stiffness, dispersion is more or less the same whatever the rotation speed may be.

Case 2 shows an interesting evolution of dispersion along with rotation speed. Uncertainty being about the first disc density, it impacts gyroscopic effects that depend on rotation speed $\Omega$. This implies that consequences of this uncertainty on eigenmodes evolve with the rotation speed. Sets 1 and 2 of Figure $10(b)$ show that it creates an obvious decrease of dispersion on mode $3 \mathrm{~B}$ along with an increase on mode $2 \mathrm{~F}$ when the rotation speed increases.

Finally, the more realistic case 3 with lower uncertainties on inputs also presents great eigenvalue variations (sets 1 and 2 of Fig. 10(c)), displaying up to $10 \mathrm{~Hz}$ between the lowest and the highest evaluated critical speed for the third forward mode (Fig. 11(c)) that is up to $20 \%$ of the deterministic value. This emphasizes the need for being able to conduct studies for uncertain structure dimensioning.

This set of results points out two things: first, comparison of the first and second cases brings to the fore that variation of the uncertainty impact with respect to rotation speed highly differs depending on the uncertainty location. Different evolutions can be observed for each studied case for eigenvalues and subsequently, for critical speeds. Second, the third processed case shows that slight modifications of the structure can lead to great variations of its modal properties which are key to dimensioning. This emphasizes the usefulness of methods providing propagation of input uncertainties to modal quantities and the ability of PC expansion to do so.

3.4.2 Eigenvectors Dispersion. Figures 12, 13, and 14 expose the MAC-like representation (detailed in Sec. 3.2.1) of eigenvectors dispersion in case of a PC approximation of order 2: the first six stochastic modes studied are compared to the first 12 deterministic modes denoted $1, \ldots, 12$ and their complex conjugates, denoted $\overline{1}, \ldots, \overline{12}$.. This information is given for four different rotation speeds, $\Omega=0,20,45,70 \mathrm{~Hz}$. Each time, Figs. 12(a), 13(a), and 14(a) refer to the mean of the stochastic indicator while Fig. 12(b), 13(b), and 14(b) refer to its standard deviation.

Figure 15 represents the mean of deformed shapes at critical speed (blue line) and the mean plus or minus three times the standard deviation at each nodes.

As a general observation, one can say that for every case and every rotation speed, the mean of the deterministic mode matching the studied stochastic mode is close to 1 which is not surprising as eigenvectors are known not to vary a lot. Breaking of the diagonal at speed $\Omega=70 \mathrm{~Hz}$ comes from the mode crossing that occurs between mode $2 \mathrm{~F}$ and $3 \mathrm{~B}$ around $\Omega=67 \mathrm{~Hz}$. As deterministic modes are ordered following their eigenfrequencies, mode $2 \mathrm{~F}$ switches from deterministic mode 4 to mode 5 while mode $3 \mathrm{~B}$ switches from deterministic mode 5 to mode 4 for $\Omega>67 \mathrm{~Hz}$.

Then, comparing case 1 and case 2 standard deviations (Fig. 12(b) and Fig. 13(b)), one can see that it mostly involves the underlying deterministic mode in the first case while in the second one, the dispersion is more diffuse and spread over several deterministic modes. Combining uncertainty on both shaft stiffness and first disc density, one gets a higher dispersion on eigenvectors (Fig. 14(b)) as several deterministic modes are involved in the stochastic modes decomposition with non-negligible contributions.

Finally, many of the considered deterministic modes have a negligible contribution to the decomposition of a given stochastic eigenvector. This observation is in favor of the selection of a few deterministic modes only to project stochastic eigenvectors onto, as mentioned in Sec. 3.3. This would drastically decrease the system size and hence the computation cost.

\section{Conclusion}

A method for computing stochastic eigenvalues and eigenvectors has been presented. It is based on a Polynomial Chaos expansion with complex weights. This method then lets real and imaginary parts of eigenmodes evolve independently when uncertainty is introduced in the system. It was applied successfully to a finite element rotor model when considering three uncertain configurations. Comparison with direct Monte Carlo simulations showed that the proposed approach returns very accurate results even in the case of multiple uncertain parameters and has a great efficiency in terms of computation time. Moreover, this application demonstrated that depending on the organ which undergoes uncertainty, eigenmodes dispersion evolution along with rotation speed can be very different and needs to be taken into account for proper structure dimensioning.

\section{Acknowledgment}

The authors gratefully acknowledge the financial support of the French National Research Agency through the Young Researcher program ANR-07-JCJC-0059-01-CSD2.

\section{Appendix A: Hermite Multivariate Polynomials}

Hermite polynomials (one dimension) can be defined using a derivative

$$
h_{n}(\xi)=(-1)^{n} \mathrm{e}^{\xi^{2} / 2} \frac{\mathrm{d}^{n} \mathrm{e}^{-\xi^{2} / 2}}{\mathrm{~d} \xi^{n}}
$$

or recursively (formula from Ref. [23, Sec. 3.2]):

$$
\begin{gathered}
h_{0}(\xi)=1 \\
\frac{d h_{n}(\xi)}{d \xi}=n h_{n-1}(\xi) \\
h_{n}(0)= \begin{cases}0 & \text { if } n \text { is odd } \\
(-1)^{n / 2} \frac{n !}{2^{n / 2}\left(\frac{n}{2}\right) !} & \text { if } n \text { is even }\end{cases}
\end{gathered}
$$

The general formula for multi-dimensional Hermite polynomials is

$$
\psi_{m}\left(\xi_{i_{1}}, \ldots \xi_{i_{n}}\right)=(-1)^{n} \mathrm{e}^{\frac{1}{2} \xi^{\mathrm{T}} \xi} \frac{\partial^{n} \mathrm{e}^{-\frac{1}{2} \xi^{\mathrm{T}} \xi}}{\partial \xi_{i_{1}}, \ldots \partial \xi_{i_{n}}}
$$

but the formula used in practice to compute the multivariate Hermite polynomials is the one found in Ref. [23, Chap. 3, Sec. 3.1]: for $Q$ random variables, the multi-variate Hermite polynomials of order $d$ can be expressed as:

$$
\psi_{\alpha}=\prod_{i=1}^{Q} h_{a_{i}}\left(\xi_{i}\right)
$$

with $\alpha=\left(\alpha_{1}, \ldots, \alpha_{Q}\right) \in\{0, \ldots, d\}^{Q}$ such that $\sum_{i=1}^{Q} \alpha_{i}=d$.

There are exactly

$$
\frac{(d+Q-1) !}{d !(Q-1) !}
$$

polynomials with degree $d$. That is, for $Q$ random variables, there are $N$ polynomials with degree less than or equal to $D$ : 


$$
N=\frac{(D+Q) !}{D ! Q !}
$$

These polynomials are orthogonal with respect to the following scalar product

$$
\begin{aligned}
\langle f, g\rangle= & \frac{1}{\sqrt{2 \pi}} \int_{\xi_{1}=-\infty}^{+\infty} \ldots \int_{\xi_{Q}=-\infty}^{+\infty} f\left(\xi_{1}, \ldots \xi_{Q}\right) \\
& \times g\left(\xi_{1}, \ldots \xi_{Q}\right) e^{-\xi^{\mathrm{T}} \xi / 2} d \xi_{1}, \ldots \xi_{Q}
\end{aligned}
$$

\section{Appendix B: Finite Element Rotor Matrices}

Matrices are given for the following degrees of freedom order per each node $[v, w, \theta, \psi]^{\mathrm{T}}$ with $v$ and $w$ defining displacements in $y$ and $z$ directions respectively and $\theta=\partial v / \partial x$ and $\psi=-\partial w / \partial x$ defining rotations about $z$ and $y$ axis.

B.1 Elementary Matrices for a Shaft Beam Finite Element. These matrices are given for one beam constituting the shaft. The shaft matrices $\mathbf{M}_{s T}, \mathbf{M}_{s R}, \mathbf{G}_{s}$ and $\mathbf{K}_{s}$ are obtained by assembling the elementary matrices for the seven beam elements:

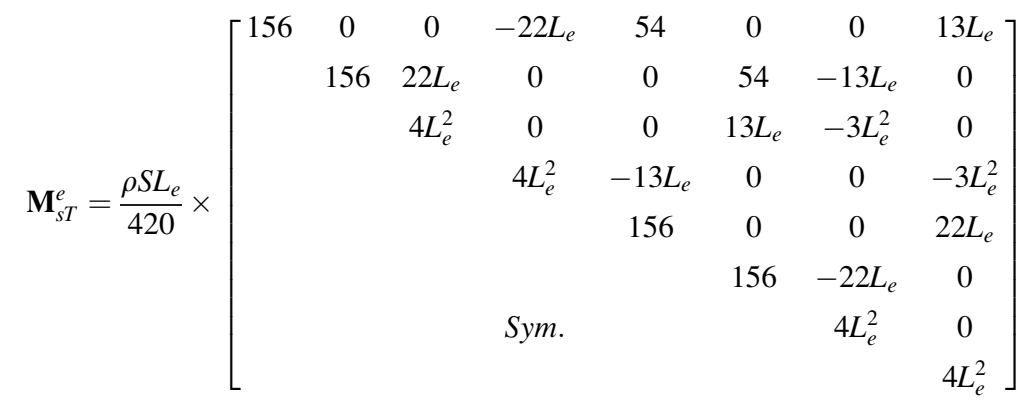

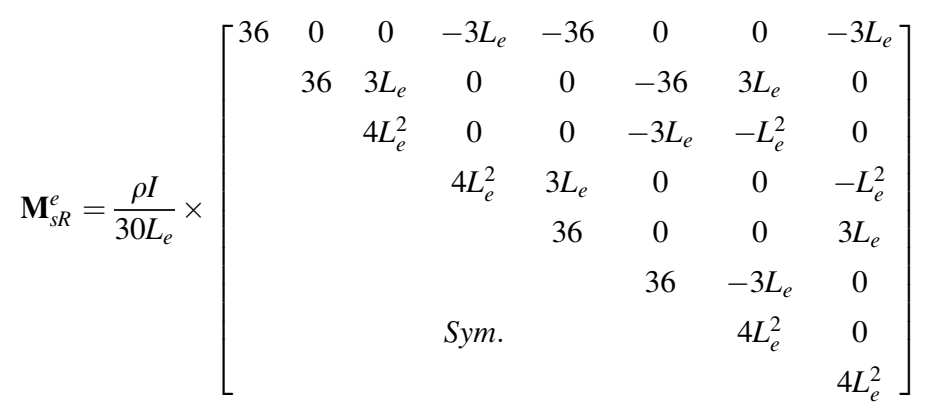

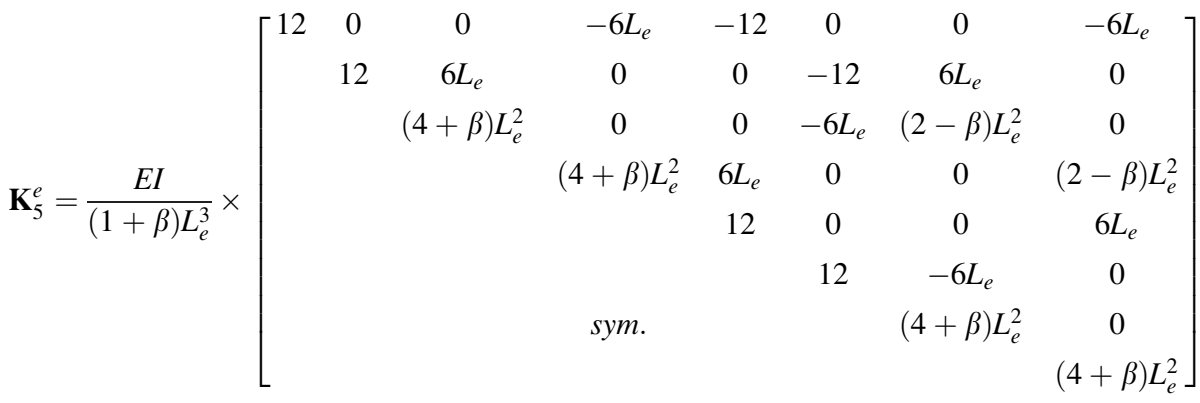

where $S$ is the area of the cross section, $I$ is the second moment of area about any axis perpendicular to the rotor axis and $\beta$ is the shear modulus $\beta=12 E I /\left(G S_{r} L_{e}^{2}\right), S_{r}$ being the reduced area of the cross section.

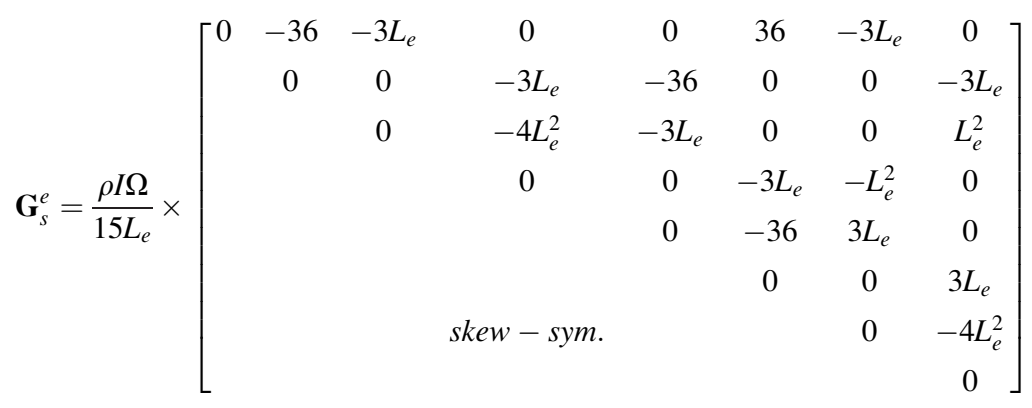




\section{B.2 Matrices for Disc $n$.}

$$
\begin{gathered}
\mathbf{M}_{d n T}=\left[\begin{array}{cccc}
m_{d n} & 0 & 0 & 0 \\
0 & m_{d n} & 0 & 0 \\
0 & 0 & 0 & 0 \\
0 & 0 & 0 & 0
\end{array}\right] \\
\mathbf{M}_{d n R}=\left[\begin{array}{cccc}
0 & 0 & 0 & 0 \\
0 & 0 & 0 & 0 \\
0 & 0 & I_{d n}^{d} & 0 \\
0 & 0 & 0 & I_{d n}^{d}
\end{array}\right] \\
\mathbf{G}_{d n}=\Omega\left[\begin{array}{cccc}
0 & 0 & 0 & 0 \\
0 & 0 & 0 & 0 \\
0 & 0 & 0 & -I_{d n}^{p} \\
0 & 0 & I_{d n}^{p} & 0
\end{array}\right]
\end{gathered}
$$

where $m_{d n}$ denotes the mass of the $n$-th disc while $I_{d n}^{p}$ and $I_{d n}^{d}$ define its polar moments of inertia about the rotor axis and about any axis perpendicular to the rotor axis, respectively.

\section{B.3 Stiffness Matrix for Bearing $n$.}

$$
\mathbf{K}_{b n}=\left[\begin{array}{llll}
k & 0 & 0 & 0 \\
0 & k & 0 & 0 \\
0 & 0 & 0 & 0 \\
0 & 0 & 0 & 0
\end{array}\right]
$$

\section{References}

[1] Ibrahim, R., 1987, "Structural Dynamics with Parameter Uncertainties," ASME Appl. Mech. Rev., 40, pp. 309-328.

[2] Manohar, C. S., and Ibrahim, R. A., 1999, "Progress in Structural Dynamics with Stochastic Parameter Variations: 1987-1998," ASME Appl. Mech. Rev., 52(5), pp. 177-197.

[3] Schuëller, G. I., 1997, "A State-of-the-Art Report on Computational Stochastic Mechanics," Probab. Eng. Mech., 12(4), pp. 197-321.

[4] Schuëller, G. I., 2001, "Computational Stochastic Mechanics - Recent Advances," Comput. Struct., 79(22-25), pp. 2225-2234.
[5] Schuëller, G., and Pradlwarter, H., 2009, "Uncertain Linear Systems in Dynamics: Retrospective and Recent Developments by Stochastic Approaches," Eng. Struct., 31(11), pp. 2507-2517.

[6] Ghanem, R., and Spanos, P., 2003, Stochastic Finite Elements: A Spectral Approach - Revised Edition, Dover Publications, New York.

[7] Xiu, D., and Karniadakis, G. E., 2002, "The Wiener-Askey Polynomial Chaos for Stochastic Differential Equations,” SIAM J. Sci. Comput., 24(2), pp. 619-644.

[8] Lucor, D., and Karniadakis, G. E., 2004, "Adaptive Generalized Polynomial Chaos for Nonlinear Random Oscillators," SIAM J. Sci. Comput., 26(2), pp. 720-735.

[9] Soize, C., 2000, "A Nonparametric Model of Random Uncertainties for Reduced Matrix Models in Structural Dynamics," Probab. Eng. Mech., 15(3), pp. 277-294.

[10] Scheidt, J., and Purkert, W., 1983, Random Eigenvalue Problems, North Holland, Amsterdam.

[11] Ghosh, D., Ghanem, R., and Red-Horse, J., 2005, "Analysis of Eigenvalues and Modal Interaction of Stochastic Systems," AIAA J., 43(10), pp. 2196-2201.

[12] Sankar, T. S., Ramu, S. A., and Ganesan, R., 1993, "Stochastic Finite Element Analysis for High Speed Rotors," ASME J. Vib. Acoust., 115(1), pp. 59-64.

[13] Murthy, R., Mignolet, M. P., and El-Shafei, A., 2010, "Nonparametric Stochastic Modeling of Uncertainty in Rotordynamics - Part I: Formulation," ASME J. Eng. Gas Turbines Power, 132(9), 092501.

[14] Murthy, R., Mignolet, M. P., and El-Shafei, A., 2010, "Nonparametric Stochastic Modeling of Uncertainty in Rotordynamics-Part Ii: Applications," ASME J. Eng. Gas Turbines Power, 132(9), 092502.

[15] Adhikari, S., 2004, "Complex Modes in Stochastic Systems," Adv. Vib.Eng., 3(1), pp. 1-11. Available at: http://engweb.swan.ac.uk/ adhikaris/fulltext/journal/ ft25.pdf.

[16] Dessombz, O., 2000, “Analyse Dynamique de Structures Comportant des Paramètres Incertains (Dynamic Analysis of Structures with Uncertain Parameters)," Ph. D. thesis, Ecole Centrale de Lyon/MEGA, France.

[17] Ghanem, R., and Ghosh, D., 2007, "Efficient Characterization of the Random Eigenvalue Problem in a Polynomial Chaos Decomposition," Int. J. Numer. Methods Eng., 72, pp. 486-504.

[18] Lalanne, M., and Ferraris, G., 1998, Rotordynamics Prediction in Engineering, 2nd ed., Wiley, New York.

[19] Sakamoto, S., and Ghanem, R., 2002, "Polynomial Chaos Decomposition for the Simulation of Non-Gaussian Nonstationary Stochastic Processes," J. Eng. Mech., 128(2), pp. 190-201.

[20] Friswell, M. I., and Mottershead, J. E., 1995, Finite Element Model Updating in Structural Dynamics, Kluwer Academic, Dordrecht.

[21] Sinou, J.-J., and Lees, A., 2007, "A Non-Linear Study of a Cracked Rotor," Eur. J. Mech. A/Solids, 26(1), pp. 152-170.

[22] Allemang, R., 2003, "The Modal Assurance Criterion-Twenty Years of Use and Abuse," Sound Vib., 37(8), pp. 14-23. Available at: http://www.sandv.com/ downloads/0308alle.pdf.

[23] Sudret, B., and Der Kiureghian, A., 2000, "Stochastic Finite Element Methods and Reliability: A State-of-the-Art Report" Technical Report, University of California, Berkeley. 